\title{
Exact decoherence dynamics of a single-mode optical field
}

\author{
Jun-Hong $\mathrm{An}^{\mathrm{a}, \mathrm{b}}$, Ye $\mathrm{Yeo}^{\mathrm{a}}$, C. H. $\mathrm{Oh}^{\mathrm{a}}$ \\ ${ }^{a}$ Centre for Quantum Technologies and Department of Physics, National University of \\ Singapore, 3 Science Drive 2, Singapore 117543, Singapore \\ ${ }^{b}$ Department of Modern Physics, Lanzhou University, Lanzhou 730000, P. R. China
}

\begin{abstract}
We apply the influence-functional method of Feynman and Vernon to the study of a single-mode optical field that interacts with an environment at zero temperature. Using the coherent-state formalism of the path integral, we derive a generalized master equation for the single-mode optical field. Our analysis explicitly shows how non-Markovian effects manifest in the exact decoherence dynamics for different environmental correlation time scales. Remarkably, when these are equal to or greater than the time scale for significant change in the system, the interplay between the backaction-induced coherent oscillation and the dissipative effect of the environment causes the non-Markovian effect to have a significant impact not only on the short-time behavior but also on the long-time steady-state behavior of the system.
\end{abstract}

Key words: Non-Markovian decoherence, Master equation, Influence functional theory

PACS: 03.65.Yz, 03.67.-a

\section{Introduction}

In realistic conditions, it is impossible to completely isolate a quantum system $S$ from its environment $E$. A proper analysis of the quantum dynamics of $S$ must therefore take into account the decoherence effect of $S$ induced by $E$. Conventional approaches to a theoretical study of the dy\section{$\mathrm{Oh})$}

Email addresses: phyaj@nus.edu.sg (Jun-Hong An), phyohch@nus.edu.sg (C. H. 
namics of such an open quantum system have treated the interactions between $S$ and $E$ perturbatively. Invoking the Born-Markov approximation, these yield approximate equations of motion such as the Redfield or master equations [1, 2, 3, 4] for the reduced system we are interested in. The Born-Markovian approximation works well when the environment correlation time $\tau_{E}$ is small compared to the time scale $\tau_{0}$ for significant change in $S$ [3, 4]. However, in the light of recent experiments (see, for instance, Refs. $[5,6,6])$, it is evident that there are many physically relevant situations where the Markovian assumption does not hold, and a non-Markovian treatment of the open system dynamics is necessary. Furthermore, there is a general interest in the fundamental theory of open quantum systems to extend the well-developed concepts and methods for Markovian dynamics to the nonMarkovian case. The development of a general description to open quantum system has thus attracted much attention lately. Some recent works exploring the non-Markovian decoherence properties of quantum systems include Refs. [8, 9, 10, 11, 12, 13, 14, 15, 16].

In this paper, we consider the exact non-Markovian decoherence dynamics of a single-mode optical field system - a basic building block in quantum communication and computation [17]. Indeed, many of the quantum information processing protocols [18, 19], especially in the field of continuous-variable quantum information processing [20], involve optical fields. In practice, the optical field inevitably interacts with surrounding environment, which always results in the decoherence of the optical field. Actually, this decoherence effect still exists even when the optical field is transmitted in an optical fiber $[21,22]$. Decoherence will undoubtedly have a detrimental influence on the performance of the protocols. It is thus very important for one to have a complete quantitative understanding of the destructive influences of the environment. Many of the current quantum optical experiments are performed at low temperatures, under vacuum condition. In this case, the main source of decoherence is the vacuum fluctuation. Many of the theoretical studies on the decoherence dynamics of an optical field to date rely on the BornMarkovian approximation [23, 24, 25, 26]. We note that, only very recently, some phenomenological models on non-Markovian decoherence dynamics of optical fields have been studied [12, 13, 14]. Based on perturbation, these may not capture all the characteristics of the exact non-Markovian dynamics. A more satisfactory theory, derived from first principles, that describes an open optical field system is thus desirable. To this end, we apply the influence-functional method of Feynman and Vernon [27, 28] to the study of 
the system $S$ of a single-mode optical field that interacts with an environment $E$, consisting of a set of harmonic oscillators at zero temperature.

The Feynman-Vernon influence functional theory allows one to derive the exact non-Markovian dynamics of $S$. In this method, the density operator of the combined system, $S$ and $E$, is expressed as a double path integral. The exact dynamics of the reduced system $S$ is then obtained by integrating this path integral over the degrees of freedom of $E$. The effective action that governs the evolution of $S$ thus consists of the free action of $S$ and an influence functional. All the environmental effects on $S$ are dynamically incorporated in the influence functional, and both the backactions from $E$ to $S$ and from $S$ to $E$ can be treated self-consistently. In our analysis, we mainly address the issues about how the non-Markovian effect takes action and what its influence on the decoherence dynamics of the optical field system in different parameter regimes is. As an explicit example, we will study the time evolution of the so-called Schrödinger cat state [18, 19] and see what an exact treatment of its decoherence dynamics would yield.

Indeed, it had been shown that non-Markovian effect shows its significant consequence on the decoherence dynamics just by a transient oscillation in short time scale [10, 12, 13, 14]. Here, we will show, besides this transient oscillation in short time scale, the non-Markovian effect can also influence the behavior of steady state in long-time scale when $\tau_{E} \simeq \tau_{0}$ or $\tau_{E}>\tau_{0}$.

Our paper is organized as follows. In Sec. II, we introduce a model of the single-mode optical field in an environment, and the coherent-state representation. In Sec. III, we present a detailed derivation of the quantum nonMarkovian master equation using influence functional theory in the coherentstate representation. Our generalized master equation has time-dependent frequency shift and decay rate, and it reduces to the general Markovian one under certain approximation. Sec. IV is devoted to a numerical study of the system decoherence dynamics for different environmental correlation time scales. In particular, the decoherence dynamics of the Schrödinger cat state [18, 19] is investigated explicitly. Finally, a summary is given in Sec. V.

\section{A single-mode optical field in a quantized radiation field}

We consider a single-mode optical field $S$ that interacted with an environment $E$. The environment, as usual, is modeled by a collection of harmonic oscillators. The total Hamiltonian governing the coupled $S$ and $E$ is given 
by [3]

$$
H=H_{S}+H_{E}+H_{I},
$$

where

$$
H_{S}=\hbar \omega_{0} a^{\dagger} a
$$

is the Hamiltonian of the free single-mode optical field $S$,

$$
H_{E}=\hbar \sum_{k} \omega_{k} b_{k}^{\dagger} b_{k}
$$

is the Hamiltonian of the environment $E$, and

$$
H_{I}=\hbar \sum_{k}\left(g_{k} a^{\dagger} b_{k}+g_{k}^{*} a b_{k}^{\dagger}\right)
$$

is a model for the system-environment interaction. $a$ and $a^{\dagger}$ are the annihilation and creation operators of the single-mode optical field $S$ with frequency $\omega_{0}$. Similarly, $b_{k}$ and $b_{k}^{\dagger}(k=1,2, \cdots)$ are the annihilation and creation operators of the $k$-th mode of the environment with frequency $\omega_{k}$. The coupling strength between $S$ and the $k$ th mode of $E$ is given by $g_{k}$. The environment is assumed to be at zero temperature initially. By this model we will address the decoherence mechanism of $S$ due to its energy exchange with the modes of the environmental vacuum fluctuation. We emphasize the model and formulation developed in the following are also applicable to many similar systems in quantum optics, for example, an optical field in a leaky cavity [3] or in an optical fiber [21, 22], and the noise effect on a nanomechanical oscillator [29].

The Hamiltonian $H$ can be expressed, in the coherent-state representation [30], in terms of

$$
H(\bar{\alpha}, \alpha, \overline{\mathbf{z}}, \mathbf{z})=\hbar\left\{\omega_{0} \bar{\alpha} \alpha+\sum_{k}\left[\omega_{k} \bar{z}_{k} z_{k}+\left(g_{k} \bar{\alpha} z_{k}+g_{k}^{*} \bar{z}_{k} \alpha\right)\right]\right\}
$$

where $\mathbf{z}$ denotes $\left(z_{1}, z_{2}, \cdots\right)$. The coherent states $|\alpha\rangle \equiv \exp \left(\alpha a^{\dagger}\right)|0\rangle$ and $\left|z_{k}\right\rangle \equiv \exp \left(z_{k} b_{k}^{\dagger}\right)\left|0_{k}\right\rangle$ are the eigenstates of $a$ and $b_{k}$ respectively: $a|\alpha\rangle=\alpha|\alpha\rangle$; $b_{k}\left|z_{k}\right\rangle=z_{k}\left|z_{k}\right\rangle$. They are nonorthogonal: $\left\langle\bar{\alpha} \mid \alpha^{\prime}\right\rangle=\exp \left(\bar{\alpha} \alpha^{\prime}\right) ;\left\langle\bar{z}_{k} \mid z_{k}^{\prime}\right\rangle=$ $\exp \left(\bar{z}_{k} z_{k}^{\prime}\right)$. And, they form overcomplete sets: $\int d \mu(\alpha)|\alpha\rangle\left\langle\bar{\alpha}\left|=1=\int d \mu\left(z_{k}\right)\right| z_{k}\right\rangle\left\langle\bar{z}_{\mathbf{k}}\right|$, with the measures $d \mu(\alpha)=\frac{\exp (-\bar{\alpha} \alpha)}{\pi} d^{2} \alpha$ and $d \mu\left(z_{k}\right)=\frac{\exp \left(-\bar{z}_{k} z_{k}\right)}{\pi} d^{2} z_{k}$. In the path integral approach, one needs to choose a convenient representation. 
It turns out that employing the coherent-state representation allows very straightforward evaluation of the path integrals. In the following section, we will use this method to derive the exact decoherence dynamics of the optical field system.

\section{Quantum non-Markovian master equation}

\subsection{The influence functional in coherent-state representation}

Suppose $\rho_{T}(t)$ describes the state of our single-mode optical field system $S$ plus the environment $E$ as a whole. This total density matrix obeys the Liouville-von Neumann equation $i \hbar \partial \rho_{T}(t) / \partial t=\left[H, \rho_{T}(t)\right]$, which gives the formal solution:

$$
\rho_{T}(t)=\exp \left(-\frac{i}{\hbar} H t\right) \rho_{T}(0) \exp \left(\frac{i}{\hbar} H t\right) .
$$

In the coherent-state representation, $\rho_{T}(t)$ can be expressed in terms of

$$
\begin{aligned}
& \left\langle\bar{\alpha}_{f}, \overline{\mathbf{z}}_{f}\left|\rho_{T}(t)\right| \alpha_{f}^{\prime}, \mathbf{z}_{f}^{\prime}\right\rangle=\int d \mu\left(\mathbf{z}_{i}\right) d \mu\left(\alpha_{i}\right) d \mu\left(\mathbf{z}_{i}^{\prime}\right) d \mu\left(\alpha_{i}^{\prime}\right) \\
& \times\left\langle\bar{\alpha}_{f}, \overline{\mathbf{z}}_{f} ; t \mid \alpha_{i}, \mathbf{z}_{i} ; 0\right\rangle\left\langle\bar{\alpha}_{i}, \overline{\mathbf{z}}_{i}\left|\rho_{T}(0)\right| \alpha_{i}^{\prime}, \mathbf{z}_{i}^{\prime}\right\rangle\left\langle\bar{\alpha}_{i}^{\prime}, \overline{\mathbf{z}}_{i}^{\prime} ; 0 \mid \alpha_{f}^{\prime}, \mathbf{z}_{f}^{\prime} ; t\right\rangle,
\end{aligned}
$$

where the resolution of identity has been used. Since we are only interested in the dynamics of $S$, it suffices to work with the reduced density matrix, which is obtained by integrating over the environmental variables. This can be expressed in terms of

$$
\begin{gathered}
\rho\left(\bar{\alpha}_{f}, \alpha_{f}^{\prime} ; t\right)=\int d \mu\left(\mathbf{z}_{f}\right)\left\langle\bar{\alpha}_{f}, \overline{\mathbf{z}}_{f}\left|\rho_{T}(t)\right| \alpha_{f}^{\prime}, \mathbf{z}_{f}\right\rangle \\
=\int d \mu\left(\alpha_{i}\right) d \mu\left(\alpha_{i}^{\prime}\right) \mathcal{J}\left(\bar{\alpha}_{f}, \alpha_{f}^{\prime} ; t \mid \bar{\alpha}_{i}, \alpha_{i}^{\prime} ; 0\right) \rho\left(\bar{\alpha}_{i}, \alpha_{i}^{\prime} ; 0\right) .
\end{gathered}
$$

Here, we have assumed that the initial total density matrix factors into a system part and an environment part, i.e., $\rho_{T}(0)=\rho(0) \otimes \rho_{E}(0)$. Now, it remains to determine the effective propagating function for the reduced density matrix,

$$
\begin{aligned}
& \mathcal{J}\left(\bar{\alpha}_{f}, \alpha_{f}^{\prime} ; t \mid \bar{\alpha}_{i}, \alpha_{i}^{\prime} ; 0\right)=\int d \mu\left(\mathbf{z}_{f}\right) d \mu\left(\mathbf{z}_{i}\right) d \mu\left(\mathbf{z}_{i}^{\prime}\right) \\
& \times\left\langle\bar{\alpha}_{f}, \overline{\mathbf{z}}_{f} ; t \mid \alpha_{i}, \mathbf{z}_{i} ; 0\right\rangle \rho_{E}\left(\overline{\mathbf{z}}_{i}, \mathbf{z}_{i}^{\prime} ; 0\right)\left\langle\bar{\alpha}_{i}^{\prime}, \overline{\mathbf{z}}_{i}^{\prime} ; 0 \mid \alpha_{f}^{\prime}, \mathbf{z}_{f} ; t\right\rangle .
\end{aligned}
$$


Equation (9) contains the forward and backward propagators of the total system. These can be expressed as path integrals. To evaluate the forward propagator $\left\langle\bar{\alpha}_{f}, \overline{\mathbf{z}}_{f} ; t \mid \alpha_{i}, \mathbf{z}_{i} ; 0\right\rangle$, one divides the time interval $t_{f}-t_{i}$ into $N$ equal subintervals. This is followed by inserting $N-1$ copies of the resolution of identity, each between a subinterval, and taking the limit of $N$ large. The path integral representation of the forward propagator can then be obtained:

$$
\left\langle\bar{\alpha}_{f}, \overline{\mathbf{z}}_{f} ; t \mid \alpha_{i}, \mathbf{z}_{i} ; 0\right\rangle=\int D^{2} \mathbf{z} D^{2} \alpha \exp \left(\frac{i}{\hbar} S[\overline{\mathbf{z}}, \mathbf{z}, \bar{\alpha}, \alpha]\right)
$$

with

$$
S[\overline{\mathbf{z}}, \mathbf{z}, \bar{\alpha}, \alpha]=S_{S}[\bar{\alpha}, \alpha]+S_{I}[\overline{\mathbf{z}}, \mathbf{z}, \bar{\alpha}, \alpha]+S_{E}[\overline{\mathbf{z}}, \mathbf{z}],
$$

where $S_{S}, S_{E}$, and $S_{I}$ are the (complex) actions corresponding to $H_{S}, H_{E}$, and $H_{I}$ respectively. All the functional integrations are evaluated over paths $\overline{\mathbf{z}}(\tau), \mathbf{z}(\tau), \bar{\alpha}(\tau)$, and $\alpha(\tau)$ with endpoints $\overline{\mathbf{z}}(t)=\overline{\mathbf{z}}_{f}, \mathbf{z}(0)=\mathbf{z}_{i}, \bar{\alpha}(t)=\alpha_{f}$, and $\alpha(0)=\alpha_{i}$. The backward propagator $\left\langle\bar{\alpha}_{i}^{\prime}, \overline{\mathbf{z}}_{i}^{\prime} ; 0 \mid \alpha_{f}^{\prime}, \mathbf{z}_{f} ; t\right\rangle$ can be evaluated in the same fashion. Substituting Eq. (10) and a similar expression for the backward propagator into Eq. (9) we obtain

$$
\begin{gathered}
\mathcal{J}\left(\bar{\alpha}_{f}, \alpha_{f}^{\prime} ; t \mid \bar{\alpha}_{i}, \alpha_{i}^{\prime} ; 0\right)=\int D^{2} \alpha D^{2} \alpha^{\prime} \exp \left\{\frac { i } { \hbar } \left(S_{S}[\bar{\alpha}, \alpha]\right.\right. \\
\left.\left.-S_{S}^{*}\left[\bar{\alpha}^{\prime}, \alpha^{\prime}\right]\right)\right\} \mathcal{F}\left[\bar{\alpha}, \alpha, \bar{\alpha}^{\prime}, \alpha^{\prime}\right]
\end{gathered}
$$

where

$$
\begin{aligned}
& \mathcal{F}\left[\bar{\alpha}, \alpha, \bar{\alpha}^{\prime}, \alpha^{\prime}\right]=\int d \mu\left(\mathbf{z}_{f}\right) d \mu\left(\mathbf{z}_{i}\right) d \mu\left(\mathbf{z}_{i}^{\prime}\right) D^{2} \mathbf{z} D^{2} \mathbf{z}^{\prime} \\
& \times \rho_{E}\left(\overline{\mathbf{z}}_{i}, \mathbf{z}_{i}^{\prime} ; 0\right) \exp \left\{\frac { i } { \hbar } \left(S_{E}[\overline{\mathbf{z}}, \mathbf{z}]+S_{I}[\overline{\mathbf{z}}, \mathbf{z}, \bar{\alpha}, \alpha]\right.\right. \\
& \left.\left.\quad-S_{E}^{*}\left[\overline{\mathbf{z}}^{\prime}, \mathbf{z}^{\prime}\right]-S_{I}^{*}\left[\overline{\mathbf{z}}^{\prime}, \mathbf{z}^{\prime}, \bar{\alpha}^{\prime}, \alpha^{\prime}\right]\right)\right\}
\end{aligned}
$$

is the influence functional containing all the environmental effects on $S$.

\subsection{Evaluation of the influence functional and effective propagating function}

Now, we calculate explicitly the influence functional of our model. Using the Feynman's procedure, one can obtain the path integral representation of the forward propagator, Eq.(10), for our system with the component actions 
as

$$
\begin{aligned}
& \left.S_{S}[\bar{\alpha}, \alpha]=-i \hbar \bar{\alpha} \alpha(t)+\int_{0}^{t} d \tau\left[i \hbar \bar{\alpha} \dot{\alpha}(\tau)-H_{S}(\bar{\alpha}, \alpha)\right]\right\} \\
& S_{E}[\overline{\mathbf{z}}, \mathbf{z}]=\sum_{k}\left\{-i \hbar \bar{z}_{k} z_{k}(t)+\int_{0}^{t} d \tau\left[i \hbar \bar{z}_{k} \dot{z}_{k}(\tau)-H_{E}(\overline{\mathbf{z}}, \mathbf{z})\right]\right\} \\
& S_{I}[\overline{\mathbf{z}}, \mathbf{z}, \bar{\alpha}, \alpha]=-\int_{0}^{t} d \tau H_{I}(\bar{\alpha}, \alpha, \overline{\mathbf{z}}, \mathbf{z}) .
\end{aligned}
$$

The path integral with respect to the environmental variables $\mathbf{z}$ can be evaluated by the saddle point method under the boundary conditions $z_{k}(0)=z_{k i}$, $\bar{z}_{k}(t)=\bar{z}_{k f}$. We have the equations of motion as

$$
\dot{z}_{k}+i \omega_{k} z_{k}=-i g_{k}^{*} \alpha, \quad \dot{\bar{z}}_{k}-i \omega_{k} \bar{z}_{k}=i g_{k} \bar{\alpha}
$$

where $\alpha$ and $\bar{\alpha}$ are treated as external sources. Substituting the solution of Eqs. (15) into Eq. (10), one can determine the desired path integral. It is noted that the prefactor under the contribution of stationary path in the coherent-state representation is equal to one, and the saddle point approach to the evaluation of the environmental part here is exact [31]. The path integral with respect to the environmental variables $\mathbf{z}^{\prime}$, in Eq. (13), can similarly be obtained. As explained in our introduction, we take the environment to be at zero temperature, i.e. $\rho_{E}\left(\overline{\mathbf{z}}_{i}, \mathbf{z}_{i}^{\prime} ; 0\right)=1$. Together with the results of the above path integrals, Eq. (13) yields the influence functional

$$
\begin{aligned}
& \mathcal{F}\left[\bar{\alpha}, \alpha, \bar{\alpha}^{\prime}, \alpha^{\prime}\right]=\exp \left\{\int _ { 0 } ^ { t } d \tau \int _ { 0 } ^ { \tau } d \tau ^ { \prime } \left[\mu ( \tau - \tau ^ { \prime } ) \left(\bar{\alpha}^{\prime}\right.\right.\right. \\
& \left.\left.-\bar{\alpha})(\tau) \alpha\left(\tau^{\prime}\right)+\mu^{*}\left(\tau-\tau^{\prime}\right) \bar{\alpha}^{\prime}\left(\tau^{\prime}\right)\left(\alpha-\alpha^{\prime}\right)(\tau)\right]\right\}
\end{aligned}
$$

with $\mu(x) \equiv \sum_{k} e^{-i \omega_{k} x}\left|g_{k}\right|^{2}$. Here, we have repeatedly used the Gaussian integral identity $\int \frac{d^{2} z}{\pi} e^{-\gamma \bar{z} z+\delta z} f(\bar{z})=\frac{1}{\gamma} f\left(\frac{\delta}{\gamma}\right)$.

In the derivation of the influence functional above, we have treated both the backactions of the environment on the system and the system on the environment self-consistently. All the effects of the environment $E$ on $S$ are collected in the influence functional, which results in a correction to the action of the free single-mode optical field $S$,

$$
\mathcal{J}\left(\bar{\alpha}_{f}, \alpha_{f}^{\prime} ; t \mid \bar{\alpha}_{i}, \alpha_{i}^{\prime} ; 0\right)=\int D^{2} \alpha D^{2} \alpha^{\prime} \exp \{\bar{\alpha} \alpha(t)
$$




$$
\begin{aligned}
& +\bar{\alpha}^{\prime} \alpha^{\prime}(t)-\int_{0}^{t} d \tau\left[\bar{\alpha} \dot{\alpha}+\dot{\bar{\alpha}}^{\prime} \alpha^{\prime}+i H_{S}(\bar{\alpha}, \alpha)\right. \\
& \left.\left.+H_{S}\left(\bar{\alpha}^{\prime}, \alpha^{\prime}\right)\right]\right\} \mathcal{F}\left[\bar{\alpha}, \alpha, \bar{\alpha}^{\prime}, \alpha^{\prime}\right] .
\end{aligned}
$$

To evaluate the path integral in Eq. (17), we again employ the saddle point method and obtain the two equations of motion:

$$
\begin{aligned}
& 0=\dot{\alpha}+i \omega_{0} \alpha+\int_{0}^{\tau} d \tau^{\prime} \mu\left(\tau-\tau^{\prime}\right) \alpha\left(\tau^{\prime}\right), \\
& 0=\dot{\bar{\alpha}}^{\prime}-i \omega_{0} \bar{\alpha}^{\prime}+\int_{0}^{\tau} d \tau^{\prime} \mu^{*}\left(\tau-\tau^{\prime}\right) \bar{\alpha}^{\prime}\left(\tau^{\prime}\right),
\end{aligned}
$$

with the boundary conditions $\alpha(0)=\alpha_{i}, \bar{\alpha}^{\prime}(0)=\bar{\alpha}_{i}^{\prime}$. The integro-differential equations render the reduced dynamics non-Markovian, with the memory effect of the environment registered in the kernel that is nonlocal in time. The solution of the integro-differential equations (18) can be expressed in terms of a complex function $u(\tau)$ as

$$
\alpha(\tau)=\alpha_{i} u(\tau), \quad \bar{\alpha}^{\prime}(\tau)=\bar{\alpha}_{i}^{\prime} \bar{u}(\tau)
$$

with the boundary condition $u(0)=1$. Substituting Eqs. (19) into Eq. (17) and using Eqs. (18), we obtain the expression of the effective propagating function of the reduced system as

$$
\begin{aligned}
\mathcal{J}\left(\bar{\alpha}_{f}, \alpha_{f}^{\prime} ; t \mid \bar{\alpha}_{i}, \alpha_{i}^{\prime} ; 0\right)= & \exp \left\{\left[u \bar{\alpha}_{f} \alpha_{i}+\bar{u} \bar{\alpha}_{i}^{\prime} \alpha_{f}^{\prime}\right.\right. \\
& \left.\left.+\left(1-|u|^{2}\right) \bar{\alpha}_{i}^{\prime} \alpha_{i}\right]\right\}
\end{aligned}
$$

where the dependence of $u$ on time is not shown explicitly for abbreviation.

\subsection{The non-Markovian master equation}

Now we can derive the master equation by computing the time derivative of Eq. (8) . First, from Eq. (20), we can write down the following identities

$$
\alpha_{i} \mathcal{J}=\frac{1}{u} \frac{\delta \mathcal{J}}{\delta \bar{\alpha}_{f}}, \quad \bar{\alpha}_{i}^{\prime} \mathcal{J}=\frac{1}{\bar{u}} \frac{\delta \mathcal{J}}{\delta \alpha_{f}^{\prime}},
$$

which will be used to remove from the time derivative of $\mathcal{J}$ its dependence on $\alpha_{i}$ and $\bar{\alpha}_{i}^{\prime}$. After taking time derivative to Eq. (8) and substituting Eqs. 

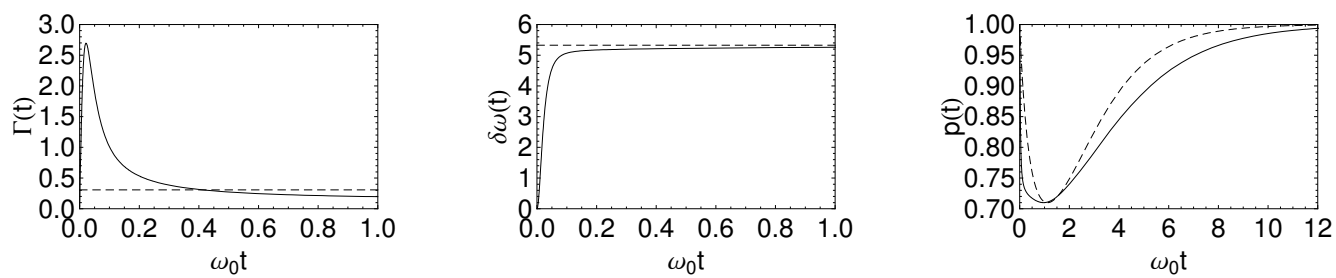

Figure 1: Comparison of the decay rate $\Gamma(t)$ (left) and the frequency shift $\delta \omega(t)$ (middle), and the purity (right) of the Schrödinger cat state between the non-Markovian (solid line) and Markovian (dashed line) results in the weak coupling and short environmental correlation time regime. The parameters $\omega_{c} / \omega_{0}=50.0, \eta=0.1$, and $\beta_{0}=1.0$ are used in the numerical calculation.
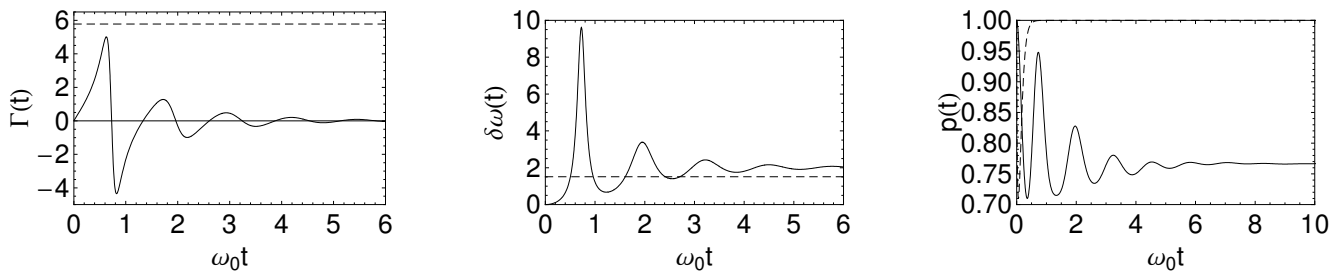

Figure 2: Comparison of the decay rate $\Gamma(t)$ (left), frequency shift $\delta \omega(t)$ (middle), and purity $p(t)$ (right) of the Schrödinger cat state between the non-Markovian (solid line) and Markovian (dashed line) results in the strong coupling and long environmental correlation time regime. The parameters $\omega_{c} / \omega_{0}=1.0, \eta=5.0$, and $\beta_{0}=1.0$ are used in the numerical calculation.
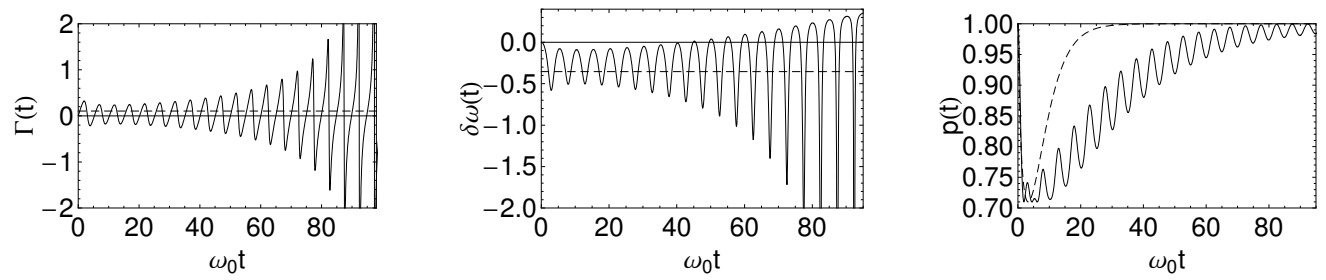

Figure 3: Comparison of the decay rate $\Gamma(t)$ (left), frequency shift $\delta \omega(t)$ (middle), and purity $p(t)$ (right) of the Schrödinger cat state between the non-Markovian (solid line) and Markovian (dashed line) results when $\tau_{E} \gg \tau_{0}$. The parameters $\omega_{c} / \omega_{0}=0.2, \eta=5.0$, and $\beta_{0}=1.0$ are used in the numerical calculation. 
(20) and (21) into it, we obtain the evolution equation

$$
\begin{aligned}
& \dot{\rho}\left(\bar{\alpha}, \alpha^{\prime} ; t\right)=\left\{-i \Omega(t)\left[\bar{\alpha} \frac{\delta \rho\left(\bar{\alpha}, \alpha^{\prime} ; t\right)}{\delta \bar{\alpha}}-\frac{\delta \rho\left(\bar{\alpha}, \alpha^{\prime} ; t\right)}{\delta \alpha} \alpha\right]\right. \\
& \left.+\Gamma(t)\left[2 \frac{\delta^{2} \rho\left(\bar{\alpha}, \alpha^{\prime} ; t\right)}{\delta \bar{\alpha} \delta \alpha}-\bar{\alpha} \frac{\delta \rho\left(\bar{\alpha}, \alpha^{\prime} ; t\right)}{\delta \bar{\alpha}}-\frac{\delta \rho(t)}{\delta \alpha} \alpha\right]\right\}
\end{aligned}
$$

where

$$
\frac{\dot{u}}{u} \equiv-\Gamma(t)-i \Omega(t)
$$

and from here on we drop the subscript $f$.

Next, we introduce the following functional differential relations in the coherent-state representation:

$$
\begin{gathered}
\bar{\alpha} \frac{\delta \rho\left(\bar{\alpha}, \alpha^{\prime} ; t\right)}{\delta \bar{\alpha}} \longleftrightarrow a^{\dagger} a \rho(t), \frac{\delta \rho\left(\bar{\alpha}, \alpha^{\prime} ; t\right)}{\delta \alpha} \alpha \longleftrightarrow \rho(t) a^{\dagger} a, \\
\frac{\delta^{2} \rho\left(\bar{\alpha}, \alpha^{\prime} ; t\right)}{\delta \bar{\alpha} \delta \alpha} \longleftrightarrow a \rho(t) a^{\dagger}
\end{gathered}
$$

with which we arrive at our final operator form of the non-Markovian master equation

$$
\dot{\rho}(t)=-\frac{i}{\hbar}\left[H^{\prime}(t), \rho(t)\right]+\Gamma(t)\left[2 a \rho(t) a^{\dagger}-a^{\dagger} a \rho(t)-\rho(t) a^{\dagger} a\right]
$$

where $H^{\prime}(t) \equiv \hbar \Omega(t) a^{\dagger} a$. This is the exact master equation for the reduced system dynamics. Our exact master equation (25) is similar to the nonMarkovian master equation of a two-level atom in a bosonic environment [32, 4]. This similarity for the fully different systems comes from the fact that both the derivations are based on the rotating wave approximation and the initial vacuum state of the environment. $\Omega(t)$, named as time-dependent Lamb shifted frequency in the two-level atom system, plays here the role of a time-dependent shifted frequency of the single-mode optical field $S$ induced by the environment $E$. $\Gamma(t)$ represents a time-dependent decay rate of the optical field. We emphasize that our derivation of the master equation goes beyond the Born-Markovian approximation and contains all the backactions between $E$ and $S$ self-consistently. It can be seen that the only difference between Eq. (25) and the master equation under Born-Markovian approximation [3] is the time-dependent coefficients. So we argue that all the non-Markovian characteristics reside in the time-dependent coefficients of the generalized master equation. 
The time-dependent coefficients in our generalized master equation, determined by Eq. (23), essentially depend on the so-called spectral density, which characterizes the coupling strength of the environment to the system with respect to the environmental frequencies. It is defined as $J(\omega) \equiv$ $\sum_{k}\left|g_{k}\right|^{2} \delta\left(\omega-\omega_{k}\right)$. In the continuous limit of the environmental frequencies, we model in our work the coupling of the optical field with the environment has a spectral density as

$$
J(\omega)=\eta \omega\left(\frac{\omega}{\omega_{c}}\right)^{n-1} e^{-\frac{\omega}{\omega_{c}}}
$$

where $\eta$ is a dimensionless coupling constant, and $\omega_{c}$ is an exponential cutoff frequency. The environment is classified into three categories [33]: sub-Ohmic if $0<n<1$, Ohmic if $n=1$, and super-Ohmic if $n>1$. Different spectral densities manifest different non-Markovian decoherence dynamics [33]. The spectral density Eq. (26) is motivated by the physical consideration that not all the modes of the environment give the same contribution to the coupling to the optical field system. Thus, the spectral density form is physically reasonable to model the decoherence of our optical field system. Actually such spectral density, especially the Ohmic one, is widely used in the the decoherence analysis of optical fields in the scenario of the continuous variable quantum information processing [10, 13, 14, 34].

Before presenting our numerical results in the next section, we show how our generalized master equation reduces to the conventional master equation by introducing the relevant Markovian approximation. By defining new dynamical variables as $x(\tau)=\alpha(\tau) e^{i \omega_{0} \tau}$, we can recast the first equation of Eqs.(18) into

$$
\dot{x}(\tau)+\int_{0}^{\infty} d \omega J(\omega) \int_{0}^{\tau} d \tau^{\prime} e^{i\left(\omega_{0}-\omega\right)\left(\tau-\tau^{\prime}\right)} x\left(\tau^{\prime}\right)=0 .
$$

Then, invoking the Markovian approximation, $x\left(\tau^{\prime}\right) \simeq x(\tau)$, namely, approximately taking the dynamical variable to be the one that depends only on the present time so that any memory effect is ignored. The Markovian approximation is mainly based on the physical assumption that the correlation time of the environment is much smaller compared with the typical time scale of the system evolution. Also under this assumption we can extend the upper limit of the $\tau^{\prime}$ integration in Eq. (27) to infinity and use the equality

$$
\lim _{\tau \rightarrow \infty} \int_{0}^{\tau} d \tau^{\prime} e^{ \pm i\left(\omega_{0}-\omega\right)\left(\tau-\tau^{\prime}\right)}=\pi \delta\left(\omega-\omega_{0}\right) \mp i \mathcal{P}\left(\frac{1}{\omega-\omega_{0}}\right)
$$


where $\mathcal{P}$ denotes the Cauchy principal value. The integro-differential equation (27) thus reduces to a linear ordinary differential equation. The solution of $x(\tau)$, as well as $\alpha(\tau)$ can then be obtained readily, which results in

$$
u(\tau)=e^{-i\left(\omega_{0}-\delta \omega\right) \tau-\pi J\left(\omega_{0}\right) \tau},
$$

with $\delta \omega=\mathcal{P} \int_{0}^{\infty} \frac{J(\omega) d \omega}{\omega-\omega_{0}}$. Using this solution, one can verify from Eq. (23) that

$$
\Gamma(t)=\pi J\left(\omega_{0}\right), \quad \Omega(t)=\omega_{0}-\delta \omega,
$$

which are precisely the coefficients in the Markovian master equation of the optical system [3].

In the next section, for definiteness, we consider Ohmic environment $E$. The characteristic time scale $\tau_{E}$ of the environmental correlation function in the Ohmic case is roughly inversely proportional to the cutoff frequency $\omega_{c}$ in Eq. (26) , i.e., $\tau_{E} \simeq 1 / \omega_{c}[35]$. The cutoff frequency $\omega_{c}$, which is originally introduced to eliminate infinities in frequency integrations, therefore also determines if the dynamics of open system $S$ is Markovian or non-Markovian. Our non-perturbatively derived exact results can allow us to explore all these possibilities.

\section{Numerical results and discussions}

To illuminate the non-Markovian decoherence dynamics of $S$, we consider the following initial state of the optical field:

$$
\rho(0)=\frac{1}{N}\left[\left|\beta_{0}\right\rangle\left\langle\beta_{0}|+|-\beta_{0}\right\rangle\left\langle-\beta_{0}|+| \beta_{0}\right\rangle\left\langle-\beta_{0}|+|-\beta_{0}\right\rangle\left\langle\beta_{0}\right|\right],
$$

where $N=2\left(e^{\left|\beta_{0}\right|^{2}}+e^{-\left|\beta_{0}\right|^{2}}\right)$ is a normalization constant. This is known as the Schrödinger cat state and has been produced experimentally [18, 19]. After some straightforward calculations, we obtain, via Eqs. (8) and (20),

$$
\begin{aligned}
\rho(t)= & \frac{1}{N}\left[e^{\left|\beta_{0}\right|^{2}-|\beta|^{2}}(|\beta\rangle\langle\bar{\beta}|+|-\beta\rangle\langle-\bar{\beta}|)\right. \\
& \left.+e^{-\left(\left|\beta_{0}\right|^{2}-|\beta|^{2}\right)}(|-\beta\rangle\langle\bar{\beta}|+| \beta\rangle\langle-\bar{\beta}|)\right],
\end{aligned}
$$

where $\beta=\beta_{0} u(t)$. From Eq. (32), the purity which is defined as $p(t)=$ $\operatorname{Tr} \rho^{2}(t)$ can be calculated readily as

$$
\begin{aligned}
p(t)= & \frac{2}{N^{2}}\left[e^{2\left|\beta_{0}\right|^{2}}+e^{-2\left|\beta_{0}\right|^{2}}+e^{2\left|\beta_{0}\right|^{2}-4|\beta|^{2}}\right. \\
& \left.+e^{-2\left|\beta_{0}\right|^{2}+4|\beta|^{2}}+4\right] .
\end{aligned}
$$


In Fig. 1, we plot the numerical results of the decay rate $\Gamma(t)$, frequency shift $\delta \omega(t)$, and purity $p(t)$ when $\tau_{E} \ll \tau_{0}$. We note that the exact $\Gamma(t)$ and $\delta \omega(t)$ differ from their corresponding values obtained via a Markovian treatment only over a very short time interval. For $t<\tau_{E}$, both coefficients grow very quickly, while for $t>\tau_{E}$, they gradually approach the corresponding Markovian results, given by Eqs. (30), as $t$ approaches $\tau_{0}$. The finite, almost steady, positive decay rate guarantees the irreversibility of the system dynamics. Accordingly, the exact time evolution of the purity shows only slight deviation from the Markovian results. The Schrödinger cat state eventually evolves to a steady state, namely the ground state of $S: \rho_{g}=|0\rangle\langle 0|$. Clearly, in this case, the backaction of the environment $E$ has negligible effect on the dynamics of system $S$, and the Markovian approximation is applicable. We say the system dynamics is mainly governed by the dissipative effect of $E$.

Figure 2 shows the decay rate $\Gamma(t)$, frequency shift $\delta \omega(t)$, and purity $p(t)$, when $\tau_{E}=\tau_{0}$. In this case, the backaction of the environment $E$ has a considerable impact on the dynamics of our system $S$, and the Markovian approximation is not applicable. Firstly, we note that, in contrast to the Markovian treatment, the decay rate can take negative values. Physically, this corresponds to $S$ reabsorbing a photon from $E$, which will lead to an increase in the photon number of $S$ [4]. Next, more interestingly, we note that the decay rate approaches zero asymptotically, which dramatically differs from the Markovian results. Consequently, the exact evolution of the Schrödinger cat state is drastically different from the Markovian results. In particular, it eventually evolves to some steady state, which is not the ground state $\rho_{g}$. The backaction of the environment causes the system to undergo transient oscillations, which is characteristic of non-Markovian dynamics. From previous studies [12, 13, 14], one would have concluded that non-Markovian effects only show up in short-time dynamics. Our results, however, clearly show on the contrary that non-Markovian effects can also have an influence on the long-time behavior of the system dynamics and the final steady state of $S$. This is because that the dissipative effect of $E$ is strongly counteracted by the effect due to the backaction of $E$.

The results under the condition $\tau_{E} \gg \tau_{0}$ is shown in Fig. 3. Because of the extremely long memory effect of the environment, the backaction from $E$ to $S$ is so strong that it governs the dynamics of $S$. This causes the decay rate to oscillate over a very long duration. This is typical of non-Markovian dynamics [10]. The 'equilibrium' position for the oscillation of $\Gamma(t)$ is not at zero, but a small positive value. The positivity of the equilibrium position 
of the decay rate oscillation makes the system dynamics experiences weak dissipation. Such weak dissipation is verified by the time evolution of the purity of the Schrödinger cat state in Fig. 3. The evolution of $p(t)$ also shows that the coherent oscillation induced by the backaction of $E$ persists for a very long time, even as the state approaches the ground state.

In summary, depending on $\tau_{E}$ in comparison to $\tau_{0}$, the decoherence dynamics of $S$ shows different behaviors. For $\tau_{E} \ll \tau_{0}$, the exact results show only slight deviation from the Markovian ones. Since the effect due to backaction of $E$ is extremely small, the system dynamics is mainly governed by the dissipative effect of $E$, and the widely used Markovian approximation is applicable. For $\tau_{E}=\tau_{0}$, the considerable backaction induced by the near resonant interaction between $E$ and $S$ counteracts the dissipative effect, and results in zero decay rate asymptotically. This causes dissipation to cease before the system decays to its ground state. That is, the steady state is no longer the ground state, unlike the previous case. When $\tau_{E} \gg \tau_{0}$, the backaction of $E$ is so strong that it governs the dynamics of $S$. The decay rate of the system oscillates about some equilibrium position over a very long period of time. The positivity of such an equilibrium position guarantees the overall weak dissipation effect of $E$.

\section{Conclusion}

In this work, using the influence-functional method of Feynman and Vernon, we investigate the exact decoherence dynamics of a single-mode optical field $S$ in an environment at zero temperature. We derive an exact generalized master equation for $S$. The equation's time-dependent coefficients depend on the environmental spectral density. These determine the exact dynamics of $S$. We conclude from our numerical results that $E$ exerts two competing influences on $S$. One effect, $\mathcal{E}_{1}$, is dissipation, and is responsible for the decoherence of $S$. The other, $\mathcal{E}_{2}$, is due to the backactions of $E$ on $S$. The backaction of $E$ on $S$ means that $E$ with its state changed due to interaction with $S$, in turn, exerts its dynamical influence back on $S$. This is the physical origin of the non-Markovian dynamics of $S$. In the conventional Born-Markovian treatment, one generally neglects the backaction of the environment. So, in that case, we do not see the effect due to backaction on the dynamics of $S$. The degree of manifestation of $\mathcal{E}_{1}$ and $\mathcal{E}_{2}$ in the dynamics of $S$ depends on $\tau_{E}$ in comparison with $\tau_{0}$. For $\tau_{E} \ll \tau_{0}, \mathcal{E}_{1}$ dominates and $\mathcal{E}_{2}$ only gives rise to a transient coherent oscillation of $S$. The state of $S$ evolves 
to the ground state $\rho_{g}$, which is coincident with the Markovian result. If $\tau_{E}=\tau_{0}, \mathcal{E}_{1}$ and $\mathcal{E}_{2}$ are comparable and their effects counteract each other. The state of $S$ asymptotically evolves to some steady state, which is not the ground state of $S$. Finally, when $\tau_{E} \gg \tau_{0}, \mathcal{E}_{2}$ dominates and governs the dynamics of $S$. The state of $S$ eventually approaches to the ground state but never quite reach it.

The theory we have established is a non-perturbative description of the exact decoherence dynamics of a single-mode optical field. It is a generalization of the well-developed Born-Markovian treatment of such system. It can serve as a useful basic theoretical model in analyzing the non-Markovian decoherence dynamics of optical fields employed in practical quantum information processing schemes. It should be noted that although only the Ohmic spectral density is considered here, it is straightforward to generalize our discussion to the non-Ohmic cases.

\section{Acknowledgement}

The work is supported by NUS Research Grant No. R-144-000-189-305. J.H.A. also thanks the financial support of the NNSF of China under Grant No 10604025, and the Fundamental Research Fund for Physics and Mathematics of Lanzhou University under Grant No Lzu05-02.

\section{References}

[1] A. G. Redfield, Adv. Magn. Reson. 1 (1965) 1.

[2] G. Lindblad, Commun. Math. Phys. 48 (1976) 119.

[3] H. J. Carmichael, An Open Systems Approach to Quantum Optics, Lecture Notes in Physics, Vol. m18 (Springer-Verlag, Berlin, 1993).

[4] H.-P. Breuer and F. Petruccione, The theory of open quantum systems (Oxford University Press, Oxford, 2002).

[5] F. Dubin, D. Rotter, M. Mukherjee, C. Russo, J. Eschner, and R. Blatt, Phys. Rev. Lett. 98 (2007) 183003.

[6] F. H. L. Koppens, D. Klauser, W. A. Coish, K. C. Nowack, L. P. Kouwenhoven, D. Loss, and L. M. K. Vandersypen, Phys. Rev. Lett. 99 (2007) 106803. 
[7] D. Mogilevtsev, A. P. Nisovtsev, S. Kilin, S. B. Cavalcanti, H. S. Brandi, and L. E. Oliveira, Phys. Rev. Lett. 100 (2008) 017401.

[8] B. L. Hu, J. P. Paz, and Y. Zhang, Phys. Rev. D 45 (1992) 2843.

[9] S. Maniscalco, J. Piilo, F. Intravaia, F. Petruccione, and A. Messina, Phys. Rev. A 69 (2004) 052101.

[10] S. Maniscalco, S. Olivares, and M. G. A. Paris, Phys. Rev. A 75 (2007) 062119 .

[11] C.-H. Chou, T. Yu, and B. L. Hu, Phys. Rev. E 77 (2008) 011112.

[12] M. Ban, J. Phy. A: Math. Gen. 39 (2006) 1927.

[13] K.-L. Liu and H.-S. Goan, Phys. Rev. A 76 (2007) 022312.

[14] J.-H. An and W. M. Zhang, Phys. Rev. A 76 (2007) 042127.

[15] B. Bellomo, R. Lo Franco, and G. Compagno, Phys. Rev. Lett. 99 (2007) 160502 .

[16] H.-P. Breuer, Phys. Rev. A 75 (2007) 022103.

[17] M. A. Nielsen and I. L. Chuang, Quantum Computation and Quantum Information (Cambridge University Press, Cambridge, 2000).

[18] A. Ourjoumtsev, R. Tualle-Brouri, J. Laurat, and P. Grangier, Science $312(2006) 83$.

[19] A. Ourjoumtsev, H. Jeong, R. Tualle-Brouri, and P. Grangier, Nature (London) 448 (2007) 784.

[20] S. L. Braunstein and P. v. Loock, Rev. Mod. Phys. 77 (2005) 513.

[21] T. Pellizzari, Phys. Rev. Lett. 79 (1997) 5242.

[22] A. Biswas and D. A. Lidar, Phys. Rev. A 74 (2006) 062303.

[23] M. G. A. Paris, F. Illuminati, A. Serafini, and S. DeSiena, Phys. Rev. A 68 (2003) 012314.

[24] J. S. Prauzner-Bechcicki, J. Phys. A: Math. Gen. 37 (2004) L173. 
[25] J.-H. An, S.-J. Wang, and H.-G. Luo, J. Phys. A: Math. Gen. 38 (2005) 3579 .

[26] R. Rossi Jr., A. R. Bosco de Magalhães, M. C. Nemes, Physica A 365 (2006) 402.

[27] R. P. Feynman and F. L. Vernon, Ann. Phys. (N.Y.) 24 (1963) 118.

[28] A. O. Caldeira and A. J. Leggett, Physic A 121 (1983) 587.

[29] I. Wilson-Rae, Phys. Rev. B 77 (2008) 245418.

[30] W. M. Zhang, D. H. Feng, and R. Gilmore, Rev. Mod. Phys. 62 (1990) 867.

[31] J. R. Klauder, Phys. Rev. D 19 (1979) 2349.

[32] B. M. Garraway, Phys. Rev. A 55 (1997) 2290.

[33] A. J. Leggett, S. Chakravarty, A. T. Dorsey, M. P. A. Fisher, A. Garg, and W. Zwerger, Rev. Mod. Phys. 59 (1987) 1.

[34] C. Hörhammer and H Büttner, J. Phys. A: Math. Theor. 41 (2008) 265301.

[35] U. Weiss, Quantum Dissipative Systems, 2nd ed. (World Scientific, Singapore, 1999). 